\title{
Neuroethical issues in times of health crisis. What roles for neurosciences, IA, neurotechnologies?
}

"Respect for the human person is based on his or her irreplaceable character."

Blaise Pascal

Laure Tabouy, $\mathrm{PhD} 1^{2,3}$

Affiliations:

1Paris University, Faculty of Medicine, Paris, France

${ }^{2 H u m a n}$ Technology Foundation, 75008 Paris, France

3MaatPharma Biotech, 69007 Lyon, France

Address correspondence to:

Laure Tabouy, PhD Neuroscientist,

Scientist Project Manager and Team manager, Human Technology Foundation

E-mail: laure.tabouy@optictechnology.org; Phone: +33-6-81918625

And

Scientist preclinical project manager

MaatPharma Biotech, Lyon, France

E-mail: laure.tabouy@gmail.com 


\begin{abstract}
How can neuroscience help everyone to live and care for our confined brains? Understanding and studying human behaviour allows us to measure the impact of such confinement on each of us and to identify those who need help. The challenges are to understand the psychological repercussions following confinement and to understand how neuroscience and neurotechnology can be very interesting tools for dealing with the health crisis. Neuroscience is changing our traditional philosophical and ethical views by providing information about the biological basis of our moral behaviour. The exception of the brain is at the heart of neuroethical thinking and discourse. Neuroethics is an interdisciplinary discipline placed at the intersection between the human sciences and the neurosciences, and aims to help understand how knowledge and research in neurosciences and neurotechnologies will affect the future of society, their impact on humans, relationships, daily life, the labour market ... but also how they can help and provide solutions and answers to the questions of citizens in a health crisis. But it's a double-edged sword, collecting and analyzing brain data in real time seems to be increasingly simple and within everyone's reach, without having the hindsight of the real meaning of these data, allowing one day to read people's thoughts, control them and manipulate them. It is becoming clear that the boundaries between medical and non-medical uses of neuroscience and neurotechnology are becoming very porous, inviting us to reflect on neuroethical issues in order to put safeguards on these uses.
\end{abstract}

\title{
Keywords
}

Neurotechnologies, neuroscience, public health, brain-machine interface, artificial intelligence, coronavirus, COVID19, personal data, neuroethics, liability, stress, social isolation, containment 


\section{Introduction}

In the face of dramatic emergency circumstances, such as what the world has been experiencing since the beginning of the health crisis, it is essential to refocus on the human, to "Take care of every man and of all mankind". At the heart of this crisis, the stakes are as much social as they are medical. The coronavirus feeds on physical fragility and increases social, psychological and spiritual fragility and isolation. It first reaches the most precarious and creates new precariousness linked to the economic crisis. At a time when technologies and artificial intelligence make up our modern world and are taking more and more space to the detriment of the human being, ethics and deontology represent the best way to put man back at the center of the game. Reflections on bioethical and neuroethical issues will make it possible to take an adjusted turn, centred on benevolence and the common good, and to reflect on respect for values and human intelligence.

Being confined? What a torture for the human brain. It is not in the predisposition of the human being to remain immobile at home, to remain without movement, but to build himself and evolve in contact with his fellow men. The health crisis of Covid-19 creates stress and frustration while allowing the development of an essential capacity, inhibition [1]. Our brain is configured to move, escape, explore, discover, promoting neurogenesis and the evolution of neuronal networks. When we move, the hippocampus, called the "internal GPS", activates the striatum, which is one of the pleasure centers, and sounds pleasure [2]. It is rooted in man, a genetic inheritance of human evolution. It nourishes his spirit of discovery and travel, stimulating neural plasticity, learning and memorization.

The sanitary crisis forces the reference frames that feed this seahorse-striatum cabling to be reconfigured, in order to find other ways to satisfy it to explore and discover. Containment is a kind of life lesson. Returning to contact with our fellow citizens after this period of confinement is not easy. Overcoming this ordeal will only be possible if we are warned of the difficulties the human brain faces during this health crisis. We must take care of it and find a balance between the neuronal wiring that makes us want to go out and the need to limit travel and contact for reasons of health and ecological crises.

Neurosciences and behavioural sciences [3], supported by neurotechnologies [4], and artificial intelligence (AI) [5], [6], help anticipate the deconfinement of a population in a progressive manner by making it possible to take care of those who will need it.

The development of neurosciences and their trivialization, giving the impression that we can have access to thought, risks fuelling fantasies. The neuroethical issues that are now 
emerging [7], [8], [9] must invite us to reflect on how to adopt a more acute ethical vigilance and to remain prudent.

Reflecting on bioethical [10] and neuroethical issues in times of containment / deconfinement allows and will allow us to live this crisis in an adjusted way, centred on benevolence and the common good, on the need to take care of ourselves and the most vulnerable, and to redefine our personal space / time.

These neuroethical reflections make it possible to adjust neuroscience research and developments in neurotechnology and to create safeguards for these applications, which go far beyond the framework of medicine. The boundaries between medical and non-medical uses are becoming very porous, with commercial applications aimed at the healthy general public, necessarily involving different forms of regulation and oversight. It is also very easy to envisage military, cybersecurity applications.

The neuroethical contours and issues imposed by the health crisis have yet to be defined, formulated and adjusted. This very young discipline, officially born in 2002 [11], is structured through international projects and consortiums, such as the International Brain Initiative [12]' [13]. The problems of the effects of containment and deconfinement on the mental health of citizens show the extent to which we need to reflect on neuroethical issues. Taking care of our brains is becoming paramount and is a public health issue. As advances in the neurosciences and cognitive sciences expand, the global impact of these discoveries on health, the legal system, national and international security and defense, and consumerism increases. The issues that drive neuroethics concern the way in which neuroscience is defined, developed and conducted. But also the way in which discoveries can be interpreted and translated into the lives of individuals acting in society.

\section{Being confined brains?}

Our brain wants something new, discovery, movement, it evolves through social interactions, which seems to be what has allowed man to have a developed brain. A stimulating environment, just like physical activity, promotes neurogenesis, neuronal and synaptic plasticity [14]. They are crucial properties of the nervous system allowing it to adapt and evolve [15]' [16], using reward, pleasure and addiction circuits [17], emotions [18], stress and anxiety axis [19].

Isolation, loneliness, uncertainty, social distancing and difficulties in measuring events and understanding the seriousness of the situation have long-term psychological effects. 
Restrictions on movement and freedom have altered social ties and disrupted daily routines. Survival in the health crisis depends on the ability to adapt to these changes.

It is likely that we feel differently as time goes by, we may find it difficult to sleep, to reorganize our time, to prioritize things. Our brain has to adapt very quickly to this change of rhythm of life, to which we are not at all accustomed. We need to redefine the normality of our lives. Our synaptic connections, which are used to adapting to stimulating environments, have to reorganize themselves. As a result, our concentration, calmness, efficiency, and attention are under severe strain. We feel and live an ambivalence between remaining productive on the one hand and taking advantage of this time of unexpected introspection on the other, which can be a source of exhaustion and frustration.

Just like not knowing. The human brain needs to know what the future will be made of, and the uncertainty of a tomorrow [21] is one of the most difficult aspects to live with. The unknown is frightening, as is the lack of temporal reference points and elements to organize one's actions. Our brain is plastic and adaptable to change, but it is possible that the uncertainty we are currently experiencing about our work or our relationships may leave traces in our neural network and that our behaviours and our vision of the world may change.

The progressive confinement and deconfinement, with the social distancing that is imposed on us, are real psychological and physical trials [3]' [22]. They can be experienced as extreme situations that can provoke post-traumatic stress syndromes and lead to states of anxiety, stress, depression or even very serious addictions [23], [24], [25].

This health crisis makes us experience emotions such as fear, anxiety, anger, worry, lack of motivation and concentration, or emotional exhaustion that can be devastating. It makes us experience the power of silence on our brain [26] which acts on neurogenesis at the hippocampus level, affects the functioning of our brain, and disrupts the dialogue between the prefrontal cortex, the hippocampus and the striatum.

Stressors such as the duration of containment, lack of clarity and transparency on the severity of the crisis, lack of guidelines, uncertainties in the information given, or financial or labour repercussions may aggravate these effects. Behavioural changes may be associated with increased addictions, changes in eating behaviour, avoidance of closed and overcrowded places, the development of OCD, or feelings of fear of others.

Neuroscience is one of the disciplines at the heart of the health crisis that can help us understand and experience it. It encompasses all areas of study of the nervous system, from fundamental to more functional aspects. They were born out of the convergence of molecular and cellular approaches to nervous tissue with more integrated, neurophysiological or cognitive 
research. Its rapid development makes it possible to observe the brain without damaging it and to perform certain interventions with great precision. They involve our conception of ourselves as responsible people with free will. They affect our understanding of everyday behaviour [27].

The many advances, which go hand in hand with the development of certain exploratory techniques, including neurotechnologies and brain imaging [28], are making it possible to better unravel the mystery of the brain. It has become possible to observe that the brain is not a fixed structure, that the connections between neural cells are organized in time and space in the different brain regions and are constantly reorganized according to life experiences, learning and the environment that makes the brain unique.

This ability to change our behaviours and modify our habits translates into molecular neuroscience by involving the reward, emotion, addiction, pleasure, stress and anxiety pathways and has repercussions on the release of neurotransmitters and hormones such as dopamine, serotonin, GABA or cortisol. Certain areas of the brain, such as the limbic system, the ventral tegmental area (VTA), the striatum, the amygdala, the gonadotropic axis and the prefrontal cortex are vital and play a key role in how the brain manages this crisis [29].

Since this containment / deconfinement are imposed collective actions, but necessary to "save lives", they offer a wonderful way to exercise our capacity for inhibition [1]. It forces us to literally restrain the part of our brain that wants to explore, move, discover, which can lead to frustration and stress. Lack of human contact due to confinement leads to an increase in cortisol and a decrease in oxytocin [30].

This health crisis is a unique opportunity for self-control. During the confinement our individual and collective inhibition was protective against covid-19: not going out, not shaking hands, kissing, hugging or just being with friends was counter-intuitive but it protected us.

The isolation experienced by confinement forces the striatum to be silenced, which can no longer satisfy the desires for pleasure, food, comfort and shopping. The prefrontal cortex then takes over. The prefrontal cortex plays a role in the regulation of mood, emotional responses and personality [31]. It works at full speed, builds muscle, consolidates itself and consumes a lot of energy for this inhibition to be effective. It allows it to adjust to change by implementing a new behaviour and by programming the non-execution of certain functions. The working memory allows to keep in memory the instructions in the course of inhibition, such as barrier gestures, social distancing, wearing of the mask, markings on the ground, gloves.

This inhibition, which is continuously put in place during deconfinement, the flexibility of the prefrontal cortex allows us to counteract our egocentrism, and this is good for cognitive, social and emotional development. By withdrawing our point of view to pay attention to that of 
others, it is easier for us to commit ourselves, to put ourselves at the service of others, to put in place ideas to feel useful in times of crisis.

But in the long term, there is a risk in fighting the ATC by force of will and the prefrontal cortex. After a while, the force of will is exhausted and one gives free rein to one's desires, without limit, there is then a very great risk of addiction. Dopamine is a neurotransmitter synthesized among other things by the dopaminergic neurons of the ATV, which project themselves in particular onto the striatum. It is at the heart of the so-called reward system, involved in motivation, pleasure, dependence, addiction, and certain psychiatric disorders [32], [33]. This dopaminergic neuron system plays a role in the feeling that our actions have a meaning, a usefulness, an impact on the real world. Without this feeling, it is difficult for us to take action in the long term. Action without effect is unbearable for the human mind. An action followed by a reward tends to strengthen. The severity of depression is associated with the degree to which the latter declines in activity [34]. The dialogue between the prefrontal cortex and the amygdala plays a role in the reaction of certain emotions and the regulation of stress, in the choice of behavior by evaluating different options, and in making a link between emotions and the meaning of things [35].

The sudden stop of the world reveals the need for mental and inner exploration. Making our prefrontal cortex function regularly so that it becomes muscular and develops gives man the ability to travel through thought, to mentally explore other universes, other ideas, to be moved internally. This capacity of self-control, of inhibition is generally under-exploited in our consumer society, where everything is quickly at our disposal.

This social fast [36] is for all a traumatic event, generating stress, anxiety, fear for the future, because the health crisis is not reassuring. It can also be a time of pause and beneficial introspection [37], [38], in particular to taste silence, which has regenerative powers for our brain. Silence promotes neurogenesis in the hippocampus [26]. The brain, through its neuronal and synaptic plasticity, arms itself and adapts itself to this new living environment, to these changes, to this new context. Neurons occupy the space freed up in our heads by silence. Conversely, noise can increase stress, cortisol levels, cardiac risks, the occurrence of states of anxiety and anguish, and alter the capacity for memorization, learning, concentration and attention [39].

Neural wiring varies from person to person, which explains why we are not all equally equipped to endure and manage uncertainty, silence and loneliness. Our ability to tolerate them is a characteristic of our personality. For extroverted people, this confinement is an escape from their comfort zone. Not being in contact with others is a source of anxiety. It is these people 
who offer social connections. While introverted people are in their comfort zone. For them, seeing the world slowdown was a breath of fresh air, a breath of fresh air. The opportunity to stay at home does not scare them, on the contrary, it recharges their batteries that way.

On the other hand, the psychological fragilities already existing in some people and/or not already taken care of can be dramatically amplified. People suffering from mental disorders such as anxiety may have difficulty receiving soothing feedback from the frontal cortex in times of stress [40], [41].

Deconfinement is certainly synonymous for some people with a return to a comfort zone, a "comfort" that they will find in the street, in crowds, at parties, at dinners ..., we are all subject to post-traumatic stress disorder (PTSD), to forms of depression, to sleep disturbances, to fear of going outside. Apprehension, anxiety and rejection of others may be felt. Just like not regaining sufficient energy to achieve goals, to restart professional / personal activities. The feeling of being out of step with those who seem to have not lost the rhythm can be disturbing. This mechanism is called the Behavioural/Social Immune System [42].

A recent study [43] revealed that neural connections emanating from the hippocampus during stressful situations reached not only areas of the brain associated with physiological responses to stress, but also the frontal-lateral-dorsal cortex, an area of the brain involved in higher cognitive functions and emotion regulation. This study also revealed that when the neural connections between the hippocampus and the frontal cortex were stronger, subjects felt less stressed by images. While subjects who reported feeling more stressed, the neural network between the hippocampus and hypothalamus was more active.

What scares, traumatizes, anxiety after this confinement differs from person to person. The deconfinement phase is at risk for irrational, unplanned, unanticipated and addictive behaviour. There is a real fear of becoming useless and unfit for the world that emerges in some people, reflecting a concern about a certain "human obsolescence". It will have taken only a virus and confinement to bring down our values of use.

Finally, several recent studies suggest that Covid-19 may affect the central nervous system, such as loss of taste and smell or even disorientation [44], [45], [46]. At present, it is difficult to say whether there will be long-term repercussions on the behaviour of patients who have had these symptoms [47], [48], [49]. It is even more difficult to say whether the neurological symptoms of Covid-19 and the psychological repercussions of confinement.

It is important to better understand the link between the neurobiological and psychological consequences of confinement, the effects of isolation, loneliness, lack of 
interaction. Confinement teaches us that contact is essential for the health of our cognitive functions.

For two months, the brain has been asked to adapt to an extraordinary situation, how will it compensate for this lack? The future seems to get brighter for some, and darker for others. It is an opportunity for all of us to think about what we need. Several situations are conceivable after this social fast. Either it can lead to a great thirst for relationships where reunions, collective moments, will multiply. Or this period will have taught us to measure the importance of our links, and therefore to become more gourmet. We will taste rarity, quality rather than profusion.

The return to noise, to the noisy world will reinforce the phenomenon of habituation, impacting our cognitive functions without us noticing it, because it is integrated at our basic sensory level, and reinforcing the fear of silence. Unless we decide to be the masters of it. And to be silent regularly to make room for the neurons.

\section{What can neuroethics do in this health crisis?}

What is "returning to normal life" in a society worried about its future? Confinement is by definition a constraint in space and a destructuring in time, so it is essential to restructure things anew and give a framework to deconfinement. Coming back into contact with our fellow citizens is not easy after a period of confinement. We have to reinvent our relationships, to accept facing a life turned upside down and the uncertainty of a tomorrow that is difficult to predict. We need to become aware of personal resistance in order to make coherent and constructive decisions. The fear of becoming useless and unfit for the emerging world, reflecting a concern about a certain "human obsolescence" can be a source of anxiety. Taking care of our brain and mental health is crucial. There are many issues that are competing with each other.

Cognitive sciences and neurosciences are indispensable for understanding the health crisis and contributing to the development of adapted, simple and intelligible information, enabling the general public to understand and be able to act accordingly, in an appropriate manner, and to have confidence in this information.

What happens in our brains when we reason morally and make decisions after the confinement, we have just experienced?

Can neuroscience provide valid and viable measures and standards of psychosocial "normality" and "abnormality"? Can brain data from the neurosciences be used as a basis for guiding our individual and societal behaviour?

How might neuroscientific knowledge about the concept of free will affect issues of legal liability? How will neurosciences and neurotechnologies be used in the 21 st century on 
the global stage to influence human health and capacity and evoke the study of political and economic power interventions?

Could studies on the supposed neural bases of moral thought and action: 1) Establish trends to engage these mechanisms in the ethical practices of neuro and psychiatry? 2) Establish a basis for initiatives with social, legal, and political aims to modify cognition and moral behaviour?

At the heart of this health crisis, bioethical issues can only be worked on in the light of the complexity of living beings in order to understand and respect human beings in their vulnerability.

Neuroscience operates within the field of ethics [50]. The exception of the brain is at the heart of neuroethical thinking and discourse. They modify our traditional philosophical and ethical conceptions by providing information on the biological basis of our moral behaviour. Living beings oscillate between robustness and vulnerability, between rigidity and malleability. The robustness of living beings defines their ability to maintain themselves and to be influenced in the face of disturbances linked to their environment. This capacity gives it plasticity and adaptability.

That is what we are going through in this health crisis. We are experiencing our vulnerability as human beings that we have to accept in a society that calls for a constant increase in ourselves. All it took was a virus and a confinement to make our values of use fall and a different relational world emerge in which real and virtual relationships will have to coexist. We will have to redefine vulnerability through the prism of the technological and digital world.

Neuroethics can really help us understand how neuroscience knowledge and research can help and provide solutions and answers to citizens' questions. It aims to help understand how neurosciences and neurotechnologies will affect the future, their impact on humans, relationships, the labour market ... To think about creating safeguards for these applications, which go far beyond the framework of medicine.

This discipline is particularly focused on the neurosciences and their conceptions, methods, conduct and impact on society. It is concerned with the well-being of our brain and the ethical, legal and social issues arising from it. It covers studies in the clinical and social spheres, society's reactions to the conception of the human being, investigations of cognition, emotions and behaviour. It also deals with questions of free will, responsibility, human identity, emotions, fears, human behaviour and personality, decision making and moral judgement. 
It is on all these specific points that neuroethics can help. This crisis has changed the way we act, reason and live in society. Deconfinement is a source of apprehension, the apprehension of not returning to the life we had before, of being out of step with others, of not having enough energy to achieve our goals and restart our activities. One must rebuild, reinvent, redefine, take the time to reflect, reappropriate one's projects and one's life.

The strength of neuroethics is that it is an interdisciplinary discipline that is as much scientific as it is philosophical and legal. It describes and covers a very broad field of reflection exploring the applications and implications of the participation of neuroscience in social contexts. Neuroethics focuses on the importance of ethical questions and issues related to advances in neuroscience research for the understanding of the brain and cognition. It is the constructive critical awareness of neuroscience. It provides a set of ethical, legal and philosophical tools for responsible research, helping to advance and accelerate an ethically defensible globalized neuroscience.

It is divided into two main chapters: 1) On the study of the neural bases of morality or the neurosciences of ethics [51] and 2) On the ethical issues fostered by neuroscience research [52] and its various implications and applications in clinical medicine and in the public sphere [53] or neuroscience Ethics [8]' [7]. Its singularity lies in the heterogeneity and complexity of existing, scientifically founded technologies, interventions and knowledge, and in the capacity of the individual's free will over such technologies. Its specificity is this personal link that we "sense 1) between our brain and our behaviours and 2) between our brain and what underlies our principle of individualization, autonomy, and existence as a human being recognized in his or her dignity ${ }^{1}$.

However, while there is growing enthusiasm for neuroscience, it is also a source of concern. The ambivalence of progress in the neurosciences plays a major role in the difficulty of defining neuroethics as a discipline that would be a valuable aid in the management of the health crisis.

It is essential for the research community and for neuroscientists to address and work on the underlying issues and ethical and legal concerns that guide neuroscience research across cultural divides. One of the major challenges in neuroethics is to establish a strong, two-way dialogue between researchers and the general public.

In France, it is becoming crucial to intensify and structure reflections on the ethical and legal issues of neurosciences and neurotechnologies, as is being done in the projects of the NIH Brain Initiative ${ }^{2}$ [54], Emory University, within the Emory Center for Ethics, in Australia [55]

\footnotetext{
1 Traité de Bioéthique 2018, " Neurosciences et neuroéthique ", Hervé Chneiweiss. Ed. Cairn. France.

2 http://www.ethics.emory.edu/pillars/health_sciences/neuroethics.html
} 
and South Korea [56]. An international consortium, the International Brain Initiative (IBI) [12]' [13], in which France is represented by its very active participation in the Human Brain Project [57], [58]' [59], is currently being structured.

The IBI's neuroethics working groups are responsible for identifying the objectives, goals and challenges of neuroscience, by initiating an inclusive dialogue on/with the actors of neuroethics [12]. This project, of international and global dimension, will contribute to establishing a solid basis for intercultural neuroethics and to creating a culturally sensitive community of neuroethicists. It will have to include a commitment to understanding cultural and moral values and to managing the tensions that will arise between transnational regulatory networks.

Cultural values influence how science is conducted and how science can be adapted and integrated into society [13]. To what extent are some of these cultural values morally relevant, need to be defended or can they be challenged? These values have an impact on the neuroscience agenda, the issues that should be considered as relevant to the study of the brain, how the results of neuroscience are understood, the funding of research projects. At present, however, no project has explicitly addressed cultural perspectives within these neuroethics and neuroscience issues. Culturally informed studies can be useful in generating new questions and fostering more fruitful and ethical global science. On the other hand, gaps in the understanding of the values, cultures, traditions, whether philosophical, religious, or historical, of countries and peoples lead to missed opportunities for collaboration, advancement of projects and new discoveries. This limits the sharing of results and leads to a lack of understanding of the potential and risks of neuroscience research in the more or less long term. Care must be taken, as this lack of knowledge can reinforce misguided stereotypes rather than thoughtful engagement.

It is also essential that researchers must be accountable to society for their research, whether it is fundamental or more applied, because we are talking about neuroscience, about the brain, the most enigmatic but also the most protective organ of the human body, the place of personality, thought and identity. Scientists are the guardians of their research work and they must anticipate and examine the potential impact of the use of technologies in non-clinical contexts. But it is important that these reflections are not reserved and entrusted to neuroscientists alone. Best practices in science ethics can only flourish if there are interdisciplinary mechanisms/structures that encourage scientists to do so. Neuroethics is a constructive critical awareness to imagine and focus questions, to understand the potential impact of research projects. It is a call for a more reflective neuroscience practice using the 
social sciences to examine the potential and limitations of neuroscience questions and methodologies.

The stakes here appear all the more decisive and crucial as they concern an organ that symbolizes for many people the person himself: to know the brain better is to know oneself better; and to act on it is to act on one's identity. Researchers want to unlock the elusive secrets of the human brain (Martin and Chun 2016), they want to understand human feelings and behaviour (Okano et al, 2015). It is indisputable that the weight and responsibility of researchers leads to the conduct of ethical and culturally sensitive neuroscience research.

The International Brain Initiative has listed neuroethical issues for neuroscientists to promote awareness and discussion.

I. What is the potential impact of a neuroscience model/research on individuals, society? Unintended consequences: On social stigma / self-stigmatization

II. What are the ethical standards for biological materials and data collection? And how do local standards compare to those of global collaborators?

III. What is the moral significance of the neural systems being developed in the Research labs?

IV. How brain interventions could impact or reduce independence.

V. In what contexts could a neuroscience technology/innovation be used or deployed?

These issues are flexible and adaptable, drawing on the values and cultural frameworks of each country, beyond traditional Western values. They are "constructed to challenge what we can generally assume we all know when discussing common neuroethical issues" [13].

While there is a preliminary agreement that it is important to protect and honour the privacy of individuals, the terms have different definitions in different cultures, highlighting a difference in ideas of values themselves. These questions help to identify assumptions about the importance of the cultural perspective to avoid oversimplification or stereotyping of individuals, groups and countries.

Neuroethical issues in times of health crisis are not necessarily easy to verbalize or identify. As pointed out above, the health crisis is putting the brain and our mental health to the test, and so is the dialogue between scientific researchers and philosophers, and the general public in order to bring them out. It is also important to stress that what the French are experiencing is not necessarily similar to what other countries are experiencing. These issues to be identified and worked on will have to take the imprint of French culture in all its dimensions, in research as well as in lifestyle and political reality. 


\section{Neurotechnologies of neuroethical issues to the rescue of the health crisis.}

Relationships to technology, the place of humans in the face of technology, the fear of being replaced, real and virtual relationships will have to coexist. The new man-machine and brainmachine interfaces open up a great many ethical and legal questions. A new world of relationships is emerging.

Another very important point on which reflected neuroethics is the use of neurotechnologies [4], [60] coupled or not with AI and machine learning, and neuroimaging [61]. They allow new knowledge to be acquired and have a real clinical impact, facilitating diagnosis by acting directly in the brain to repair it, by creating new neuron circuits that take over the defective circuits. They are also techniques for modifying brain functions at the interface between the brain and the machine in order to visualize, decipher and modulate brain function and pathological dysfunctions, using electrodes, EEGs, deep brain stimulation, transcranial stimulation or chemical molecules [6].

Neurotechnologies, whether or not coupled with AI and machine learning, are remarkable tools in these times of health crisis and are invading the medical sector, whose applications are taking up more and more space on a daily basis and could have many applications, such as lie detection, neuromarketing or could, in principle, also be used in forensic psychiatry [62], [63].

Their main objectives are to record and modify brain activity, opening a new era in the scientific understanding of neural circuits and allowing new methods of access to the fundamental mechanisms underlying human identification, memories, emotions, personality and, more generally, our mind. As such, they will have profound consequences for human identity and society. The field of possibilities is growing richer as studies are conducted to better understand the functioning of the brain, the ability to decode, stimulate or modify sensory perceptions, but also to improve and combine the processing power of the brain and modify behaviour.

The more the technology fits into the privacy of the brain, the greater the risk of neurosecurity. Brainjacking", via digital technologies and mobile applications currently under study and possibly through various implants, raises questions about the guarantees of effective data protection. There is also the question of early prediction of pathologies in a healthy person. Furthermore, we live in a society where information circulates so quickly that these neurotechnologies, often still experimental, reach our fellow citizens without researchers having the time to ensure that they are suitable for a life outside the research laboratory. 
This is the case of certain neurotechnologies proposed by several companies/start-ups since the beginning of the health crisis, without any rules being imposed on their use in France [64], which make it possible to detect different cognitive and affective states.

They focus on detecting stress, anxiety, emotions, attention at work, through recordings of brain activity by capturing brain waves and translating them into digital signals, which are then processed by algorithms. They offer headsets, headphones, technologies with interesting and attractive shapes for users. These portable forms make it possible to take them everywhere, which raises many questions about the monitoring of individuals but also about the continued use of these neurotechnologies. They use Bluetooth and Wi-Fi to connect to mobile applications, they are rechargeable, they have mini EEG scanners that can detect brain waves and head movements. The resulting brain data, which has yet to be fully understood by the general public in understandable language, can be recorded under any circumstance [65]. Collecting and analyzing this brain data in real time seems to be increasingly simple and accessible to everyone.

Emotiv ${ }^{\circledR}$ offers headphones, which are scanners and algorithms, and which, thanks to the data they have accumulated from previous experiments, allow to record and evaluate brain activity in real time, indicating the level of stress and distraction of the user. The future of such a process would be applicable to the world of work, to adapt working days according to the person's state during the day. These headphones obviously allow the detection of different cognitive and affective states.

Neuralink, Openwater and Neurosity, these biotechnologies develop brain-machine interfaces and provide headsets that combine brain imaging, algorithms, random access memory, an internet connection and machine learning. The objective of some of these neurotechnologies would be to act on the motor cortex, which is involved in the planning, control and execution of voluntary movements. They build "medical" devices and neural applications to measure emotions and use thoughts to interact with technologies. Blueberry has taken out its connected glasses to help relax and increase performance.

In these times of health crisis, Open Mind technologies offers its services to detect stress, anxiety, emotions. As for Facebook, the CEO dreams of developing "brain-pathy", that is to say the fact of making people communicate from brain to brain.

Dreem, for its part, develops neurotechnologies controlled via mobile applications to improve sleep. BrainCo, an American startup, primarily allows amputees to control a hand prosthesis. Not only does it allow amputees to control a hand prosthesis, they also focus on increasing performance, detecting stress, increasing attention, concentration, focus, reactivity of the user. 
Finally, NextMind, a French startup, spin off from Normal Sup', has developed a neural interface capable of decoding images in the visual cortex in real time, making simple actions possible by detecting the attention paid to an element.

From a molecular neuroscience perspective, the use of neurotechnologies, whether for medical or non-medical purposes, has the potential to change synaptic connections in the longer or shorter term. This would have a significant impact on the behaviour and other brain functions of users (such as learning, memory, concentration or attention). The excitation/inhibition balance is maintained in the brain by the dialogue between the different neurotransmitters, this balance is essential [66]' [67] and these neurotechnologies have a significant impact on the brain [68], [69], [70].

In the treatment of a pathology, the action of these neurotechnologies rebalances this balance. Initially, as conceived and developed by researchers, they promise to be revolutionary. They are already enabling people with severe motor or sensory disabilities to regain the use of their limbs, their sensitivity or the ability to communicate with the outside world. As shown by researchers in Grenoble who have developed an exoskeleton to restore mobility to quadriplegic patients [71]. They make it possible to compensate for cognitive deficiencies [72], [73] and are therefore intended for purely medical use. These spectacular results and advances validate the proof of concept of exoskeletal piloting. They allow the long-term implementation of a semiinvasive medical device to measure brain activity and to control an exoskeleton through thought. They raise questions about the consequences of such a device and pave the way for more ethical and legal thinking and commercial issues.

In a non-medical setting, their use raises many questions. Under the pretext of focusing on the detection of emotions in order to improve the daily life of users, these neurotechnologies are used by healthy people, possibly disturbing this balance. However, these uses are not insignificant, we are not equal in front of these neurotechnologies. Each individual's brain is unique, the way it reacts to electrical stimulation from a helmet placed on the brain can have long-term consequences. Being "healthy" does not mean that one is not fragile in the face of this or that pathology. Playing on the sensitivity of the excitation/inhibition balance can promote their appearance by creating a deficit of neurotransmitters. Epileptic seizures may appear, as well as deficits in attention, concentration or memory. This may promote the development of chronic depression, OCD and schizophrenia. Trying to solve a problem of anxiety, stress or simply wanting to feel "good" without the advice of neuroscientists and doctors beforehand could be catastrophic. 
However, behind this experimental research on neuroscience and neurotechnology, questions will eventually arise about the commercialization of such devices, as well as the use and applications of these neurotechnologies in the military field [74]. There is therefore an urgent need to consider whether the use of neurotechnologies that stimulate brain activity in a period of health crisis, when the brain is already under severe strain, would not be counterproductive for some people.

Furthermore, it is difficult to say whether the symptoms observed in people with Covid19, such as loss of taste and smell or even disorientation [44], [45], [46] will have long-term repercussions on patient behaviour. The neurological symptoms of Covid-19 and the psychological repercussions of confinement are even more difficult to assess [47], [48], [49]. It would be interesting and even important to ask whether the use of these neurotechnologies a source of aggravation of neurological symptoms in people could be who have been infested with Covid-19.

It is clear today that neuroscience, neurotechnology and artificial intelligence are on the verge of making it possible, in the not too distant future, to read thoughts and control them, to manipulate people to make decisions in an insidious way. It is important to note that some artificial intelligence (AI) is part of neurotechnologies [75], [6], in this case, AI is integrated into neuroethical issues and does not raise the same problems as, for example, the AI of Facebook or Google. AI is not a human intelligence. It corresponds to a form of computational intelligence that can have access to a large amount of data but not to an understanding of the living, which combines emotion and reason. The relationship between the study of biological attention and its use as a tool to improve artificial neural networks is not always clear [76].

In other words, neuroethics includes neurotechnologies without and with AI [77]' [78].

As we can see, neurotechnology and AI can be used for both medical and non-medical purposes. However, it should be noted that they are often originally designed for one purpose and then move on to another. On the one hand, neurotechnologies were initially thought for medical use and are now increasingly being used for non-medical purposes (anxiety management, emotional state, etc.). On the other hand, artificial intelligence was first conceived from a non-medical perspective and is now used for health purposes (management of the epidemic). This shift from one purpose to another is insidious. And the most serious thing remains the fact that, because the technology already exists and is already in use, there is no longer any real question of purpose. 


\section{Neuroethics and Neurolaw ... where are we in France and Europe?}

Not everything is testable, not everything is desirable ... or even feasible. The development of innovations, technologies and neurotechnologies that have an impact on the lives of users requires ethical limits. The more we learn about the brain and how it works, the more powerful and precise neuromodulation methods and neurotechnologies become, and the more we must question the effect of these manipulations on mental states and behaviour. The incredible results obtained can no longer hide the need to formalize it. Appropriate protections of private spaces and individual identity must be integrated into our understanding of human rights. These neurotechnologies generate staggering amounts of data, which we need to understand, and their use, which can significantly alter a person's personality, thoughts and sensorimotor experience, requires attention to individual and societal protections.

Neurotechnologies, whether or not coupled with AI and machine learning, are remarkable tools and are invading the medical sector. Their applications are becoming more and more important in everyday life and could have many applications, such as the detection of stress, emotions, attention at work, neuromarketing or could, in principle, also be used in forensic psychiatry and help to increase the accuracy of risk assessment and to identify possible interventions. This is "neuro-prediction of A.I." and involves the identification of potential neurocognitive markers for the prediction of recidivism. Although it is still necessary to understand how they can be implemented [62], [63].

As knowledge of neuroscience and human behaviour expands, the moral impact of neuroscience discoveries on health and health care, legal systems, national security, consumerism, etc. is increasing....

This justifies the question of how the application of new neurotechnologies to humans should be guided and regulated. There is an urgent need for guidance.

To date in France and in Europe, there is a beginning of reflections on common guidelines to guide the development and application of these new neurotechnologies in a responsible manner. OECD recommendations of December 2019 on "responsible innovation in the field of neurotechnologies ${ }^{3 "}$ are the first international normative stones in these fields, which will enable inventors, users, researchers, and public authorities to create and use these neurotechnologies with ethical, legal and societal limits.

\footnotetext{
${ }^{3}$ https://www.oecd.org/science/recommendation-on-responsible-innovation-in-neurotechnology.htm
} 
Faced with the emergence of these ethical issues, the need to regulate neuroscientific practices has become obvious. This concern has not escaped the notice of the French legislator, who was one of the first in the world to take an interest in the problems of neuroscience. On the strength of numerous hearings and reports, ${ }^{4}$ it has enshrined a new article regulating the use of brain imaging techniques in the 2011 bioethics law ${ }^{5}$.

Even today, Article 16-14 of the Civil Code stipulates that "brain imaging techniques may only be used for medical purposes or scientific research, or in the context of judicial expertise. The express consent of the person must be obtained in writing before the examination is carried out, after he or she has been duly informed of its nature and purpose. The consent must specify the purpose of the examination. It may be revoked without formality at any time.

However, while the French legislature has perceived the urgency of addressing this issue, its approach has remained relatively narrow and timid. In fact, several issues have remained in the background. What, for example, is the risk of discrimination based on neural data $^{6}$ ? What about the possibilities of brain intervention such as neuromodulation? How can free and informed consent be ensured when the patient suffers from a mental disorder? Who is responsible for damage caused by neurotechnology? Should neurological enhancements be prohibited?

These issues first arose during the drafting of the new law on bioethics. Indeed, fed once again by numerous reports and hearings, ${ }^{7}$ the government tabled a bill on 24 July last, two of which concern the neurosciences. On the one hand, Article 12 reinforces the scope of

\footnotetext{
${ }^{4}$ PARLIAMENTARY OFFICE FOR THE EVALUATION OF SCIENTIFIC AND TECHNOLOGICAL CHOICE, Report on the evaluation of the application of Law No. 2004-800 of 6 August 2004 on bioethics, No. 1325, 17 December 2008; CENTRE D'ANALYSE STRATEGIQUE, Perspectives scientifiques et légales sur l'utilisation des sciences du cerveau dans le cadre de procédures judiciaires", Seminar of 10 December 2009; ASSEMBLEE NATIONALE, Rapport d'information fait au nom de la mission d'information sur la révision des lois de bioéthique, No. 2235, January 2010.

${ }^{5}$ Art. 45 of the law $n^{\circ} 2011-814$ of July $7,2011$.

${ }^{6}$ However, the issue has been raised in parliamentary debates. An amendment by MP Jean Léonetti (26 January 2011) proposed an article 16-15 in the Civil Code: "No one may be discriminated against on the basis of brain imaging techniques".

${ }^{7}$ PARLIAMENTARY OFFICE FOR THE EVALUATION OF SCIENTIFIC AND TECHNOLOGICAL CHOICES, The Impact and Challenges of New Technologies for Brain Exploration and Therapy, March 2012; CENTRE FOR STRATEGIC ANALYSIS, The Brain and the Law : Analysis of the Emergence of Neurolaw, Working Paper No. 2012-07, September 2012; CENTRE D'ANALYSE STRATÉGIQUE, "Le cerveau et le droit: éthique et pratique du droit neurologique", Discussion Paper No. 282, September 2012; NATIONAL ETHICS ADVISORY COMMITTEE, "Ethical Issues in Functional Neuroimaging", Opinion No. 282, September 2012. 116, 22 March 2012; NATIONAL ETHICS ADVISORY COMMITTEE, "Use of Biomedical Techniques for "Neuroimprovement" in the Non-Patient: Ethical Issues", Opinion No. 122, 12 February 2014; BIOMEDICINE AGENCY, Report on the Bioethics Law, January 2018; STATE COUNCIL, Revision of the Bioethics Law: What Options for the Future? June 2018; PARLIAMENTARY OFFICE FOR THE EVALUATION OF SCIENTIFIC AND TECHNOLOGICAL CHOICES, Report on the evaluation of the application of the law No. 2011-814 of 7 July 2011 on Bioethics, October 2018; NATIONAL ASSEMBLY, Expert mission report on the revision of the law on bioethics, January 2019; NATIONAL ADVISORY ELECTRICAL COMMITTEE, Summary report on the general states of bioethics, September 2018; NATIONAL ADVISORY ELECTRICAL COMMITTEE, "Contribution to the revision of the law on bioethics", Opinion No. 129,18 September 2018.
} 
application of the use of brain imaging in the judicial field, excluding the use of functional techniques; on the other hand, Article 13 provides for the possibility of prohibiting by decree all "acts, processes, techniques, methods and equipment intended to modify brain activity presenting a serious danger or a presumed serious danger to human health" ${ }^{8}$.

In short, legislators are increasingly seeking to regulate the use of neurotechnology. However, while one might have expected a lively discussion in the parliamentary chamber on such important issues, this was not the case, as the October debates showed ${ }^{9}$. The timid grasp of these issues by parliamentarians appears to be not so much a result of a lack of interest in the subject as a difficulty in understanding exactly what is at stake, as the subject is complex and evolving.

The European RGPD is considering similar recommendations to regulate the learning machine, CEW and personal data, generating tensions with innovators using CEW. Regulation will become indispensable, it is time for legal and ethical frameworks to be established, but this should not hinder innovation and creativity of researchers.

The International Bioethics Committee of UNESCO drafted a declaration on Covid-19 and ethical considerations on 26 March $2020 .{ }^{10}$ Chapter 9 talks about these digital technologies, including IA, and recognizes their importance in combating the pandemic, but adds that it is imperative to ensure that the ethical, legal and social issues related to their uses are 'adequately addressed'.

As can be seen, even if the legislator is seeking to better regulate the use of neurotechnologies - and indeed the new law on bioethics is a step forward in this regard - many legal questions remain unresolved. However, in our opinion, the neuroscientific community has a responsibility to enlighten the public authorities on the issues at stake in its practices, which often appear obscure, complex and too technical for the general public.

This awareness is already widely shared in the world of neuroscience. Researchers in neuroscience and neurotechnology are already trying to reflect on the use of the devices they create and that are currently being used experimentally [79], [80].

This field of neurotechnology research is evolving very rapidly and it is becoming increasingly necessary to anticipate problems in order to better prevent them.

\footnotetext{
${ }^{8}$ Draft law on bioethics adopted by the National Assembly in first reading on 15 October 2019, Adopted Text No. 343.

9 See in particular the second meeting on Friday 4 October 2019 (starting at 56.30), available at http://videos.assemblee-nationale.fr/video.8195030_5d974096db838.2eme-seance--bioethique-suite-4-octobre2019.

10 https://unesdoc.unesco.org/ark:/48223/pf0000373115.locale=fr
} 
Furthermore, because it will necessarily be revised in a few years' time, tomorrow's law on bioethics obliges us to work upstream on the issues that arise at present. It is this prospect of being on the agenda of the next law on bioethics that encourages us to engage now in this work of reflection.

In other words, there is no question here of asking researchers to restrict themselves in their research. The characteristic of the researcher is to let his or her ideas emerge and explore, to test paths, sometimes dead ends, and to change them if necessary. On the other hand, it would be appropriate for researchers to be open to these debates, to take an active part in the construction of a French neuroethical reflection, taking French culture and values as a basis.

\section{A neuroethics flight plan for the next few years? ...}

How far can and should we go? Beyond strictly ethical issues, the field of neuroethics extends to the founding philosophical questions' which the neurosciences are renewing in depth: the nature of the human being, the relationship between soul and body, free will or personal identity.

Public confidence in science should be based on responsible deployment of scientific advances. And these must be shaped by our collective moral sensitivities to ensure that such advances fit harmoniously into our culture and contribute effectively to the common good, as the consequences for human identity and society will be significant.

It is very important that citizens clearly understand, without any exaggeration, the potential benefits of these neurotechnologies, as well as their risks and limitations. This global race for technological innovation is becoming more sophisticated as the neurosciences advance and opens the door to many ethical abuses. It is undeniable that the prospect of reading thoughts and controlling them raises hopes as well as fears and calls for real caution. Attention should rather be focused on what is achievable and what is desirable to do for the common good because the consequences for human identity and society will be significant. The boundaries between medical and non-medical uses are becoming very porous, with commercial applications aimed at the healthy general public, but also military or cybersecurity applications, necessarily involving different forms of regulation and oversight.

We need neuroscience and neuroethics now. Now is when we need the neuroscientists. We need to understand how these neurotechnologies and advances in neuroscience affect the future, their impact on humans, relationships, the labour market ... What is this brain data, which is stored with the use of neurotechnologies? Do we have the right to have data from our fellow citizens, simply because they are carriers of a virus? Do we have the right to use these neurotechnologies at all costs to detect and measure stress, a person's attention at work, 
emotions, a person's well-being, by scanning brain activity without informing users of the risks and possible abuses?

In medicine, the use of technology to monitor and modify the capabilities of the human body has always been guided by a common humanistic goal: to help patients in need or, more generally, to promote, without borders, the health of the entire world population. Since the Hippocratic Oath, medicine has developed a corpus of ethical rules that have formed a deontology.

These principles of medical ethics, taught in medical schools, are closely followed by health professionals, but are also respected by society as a whole, including scientists, governments, the private sector and the military.

After World War II, modern medical ethics was institutionalized by the Belmont Report, a document written by the National Commission for the Protection of Persons in the United States. This report proposed ethical principles and guidelines for research involving human subjects based on four fundamental principles: autonomy and respect, beneficence, nonmaleficence, and justice. Issues such as informed consent, assessment of risks and benefits, and selection of subjects are addressed in a practical manner.

It would be necessary to adopt a set of French and international principles similar to the Belmont Report for neuroethics. This situation of global health crisis calls for the development of a set of clear guidelines to integrate the development of neuroscience and the use of these neurotechnologies into our fundamental societal and human values while guaranteeing the freedom of ongoing research in neuroscience.

The challenge for France is to integrate this consortium in an active way and that interdisciplinary research teams at the interface of the humanities and hard sciences can be created to work on these issues, perhaps within a new French Institute of Technology for the Humanities like those in Canada and the United States, 
1. Brand M, Wegmann E, Stark R, Müller A, Wölfling K, Robbins TW, et al. The Interaction of Person-Affect-Cognition-Execution (I-PACE) model for addictive behaviors: Update, generalization to addictive behaviors beyond internet-use disorders, and specification of the process character of addictive behaviors. Neurosci Biobehav Rev. 2019;104: 1-10. doi:10.1016/j.neubiorev.2019.06.032

2. Heller AS. Association between real-world experiential diversity and positive affect relates to hippocampal-striatal functional connectivity. Nat Neurosci. 2020. doi:https://doi.org/10.1038/s41593-020-0636-4

3. Bavel JJ Van, Baicker K, Boggio PS, Capraro V, Cichocka A, Cikara M, et al. COVID19 pandemic response. Nat Hum Behav. doi:10.1038/s41562-020-0884-z

4. Rainey S, Martin S, Christen A, Mégevand P, Fourneret E. Brain Recording, MindReading, and Neurotechnology: Ethical Issues from Consumer Devices to Brain-Based Speech Decoding. Sci Eng Ethics. 2020. doi:10.1007/s11948-020-00218-0

5. Alimadadi A, Aryal S, Manandhar I, Munroe PB, Joe B, Cheng X. Artificial intelligence and machine learning to fight covid-19. Physiol Genomics. 2020;52: 200202. doi:10.1152/physiolgenomics.00029.2020

6. Silva GA. A new frontier: The convergence of nanotechnology, brain machine interfaces, and artificial intelligence. Front Neurosci. 2018;12: 1-8. doi:10.3389/fnins.2018.00843

7. Chandler JA. Neurolaw and Neuroethics. Camb Q Healthc Ethics. 2018;27: 590-598. doi:10.1017/S0963180118000117

8. Rommelfanger KS, Jeong SJ, Montojo C, Zirlinger M. Neuroethics: Think Global. Neuron. 2019;101: 363-364. doi:10.1016/j.neuron.2019.01.041

9. Buniak L, Darragh M, Giordano J. A four-part working bibliography of neuroethics: Part 1: Overview and reviews - defining and describing the field and its practices. Philos Ethics, Humanit Med. 2014;9: 1-14. doi:10.1186/1747-5341-9-9

10. Huxtable R. COVID-19: Where is the national ethical guidance? BMC Med Ethics. 2020;21: 20-22. doi:10.1186/s12910-020-00478-2

11. Roskies A. Neuroethics for the new millenium. Neuron. 2002;35: 21-23. doi:10.1016/S0896-6273(02)00763-8

12. Adams A, Albin S, Amunts K, Asakawa T, Bernard A, Bjaalie JG, et al. International Brain Initiative: An Innovative Framework for Coordinated Global Brain Research Efforts. Neuron. 2020;105: 212-216. doi:10.1016/j.neuron.2020.01.002

13. Amadio J, Bi GQ, Boshears PF, Carter A, Devor A, Doya K, et al. Neuroethics Questions to Guide Ethical Research in the International Brain Initiatives. Neuron. 2018;100: 19-36. doi:10.1016/j.neuron.2018.09.021 
14. Frey S, Schieweck R, Forné I, Imhof A, Straub T, Popper B, et al. Physical Activity Dynamically Regulates the Hippocampal Proteome along the Dorso-Ventral Axis. Int J Mol Sci. 2020;21: 3501. doi:10.3390/ijms21103501

15. Choquet D, Triller A. The dynamic synapse. Neuron. 2013;80: 691-703. doi:10.1016/j.neuron.2013.10.013

16. Citri A, Malenka RC. Synaptic Plasticity: Multiple Forms, Functions, and Mechanisms. Neuropsychopharmacology. 2008;33: 18-41. doi:10.1038/sj.npp.1301559

17. Baik JH. Dopamine signaling in reward-related behaviors. Front Neural Circuits. 2013;7: 1-16. doi:10.3389/fncir.2013.00152

18. Janak PH, Tye KM, Sciences B, Sciences C. From circuits to behaviour in the amygdala : Nature : Nature Publishing Group. NatureCom. 2015;517: 284-292. doi:10.1038/nature14188.From

19. Daviu N, Bruchas MR, Moghaddam B, Sandi C, Beyeler A. Neurobiological links between stress and anxiety. Neurobiol Stress. 2019;11: 100191. doi:10.1016/j.ynstr.2019.100191

20. Banerjee D, Rai M. Social isolation in Covid-19: The impact of loneliness. Int J Soc Psychiatry. 2020; 20764020922269. doi:10.1177/0020764020922269

21. Peters A, McEwen BS, Friston K. Uncertainty and stress: Why it causes diseases and how it is mastered by the brain. Prog Neurobiol. 2017;156: 164-188. doi:10.1016/j.pneurobio.2017.05.004

22. West R, Michie S, Rubin GJ, Amlôt R. Applying principles of behaviour change to reduce SARS-CoV-2 transmission. Nat Hum Behav. 2020. doi:10.1038/s41562-0200887-9

23. Brooks SK, Webster RK, Smith LE, Woodland L, Wessely S, Greenberg N, et al. The psychological impact of quarantine and how to reduce it: rapid review of the evidence. Lancet. 2020;395: 912-920. doi:10.1016/S0140-6736(20)30460-8

24. Holmes EA, O'Connor RC, Perry VH, Tracey I, Wessely S, Arseneault L, et al. Multidisciplinary research priorities for the COVID-19 pandemic: a call for action for mental health science. The Lancet Psychiatry. 2020;0366: 1-14. doi:10.1016/s22150366(20)30168-1

25. Mengin A, Allé MC, Rolling J, Ligier F, Schroder C, Lalanne L, et al. Psychopathological consequences of confinement. Encephale. 2020. doi:10.1016/j.encep.2020.04.007

26. Kirste I, Nicola Z, Kronenberg G, Walker TL, Liu RC, Kempermann G. Is silence golden? Effects of auditory stimuli and their absence on adult hippocampal neurogenesis. Brain Struct Funct. 2015;220: 1221-1228. doi:10.1007/s00429-013- 
0679-3

27. Bassett DS, Sporns O. Network neuroscience. Nat Neuroscience. 2017;20: 353-364. doi:10.1038/nn.4502

28. Frewen P, Schroeter ML, Riva G, Cipresso P, Fairfield B, Padulo C, et al. Neuroimaging the consciousness of self: Review, and conceptual-methodological framework. Neurosci Biobehav Rev. 2020;112: 164-212. doi:10.1016/j.neubiorev.2020.01.023

29. Phelps EA, LeDoux JE. Contributions of the amygdala to emotion processing: From animal models to human behavior. Neuron. 2005;48: 175-187. doi:10.1016/j.neuron.2005.09.025

30. Vittner D, McGrath J, Robinson JA, Lawhon G, Cusson R, Eisenfeld L, et al. Increase in Oxytocin From Skin-to-Skin Contact Enhances Development of Parent-Infant Relationship. Biol Res Nurs. 2018;20: 54-62. doi:10.1177/1099800417735633

31. Caballero A. Mechanisms contributing to Prefrontal Cortex Maturation during Adolescence. Neurosci Biobehav Rev. 2016;70: 4-13. doi:10.1016/j.physbeh.2017.03.040

32. Schultz W. Recent advances in understanding the role of phasic dopamine activity [version 1; peer review: 3 approved]. F1000Research. 2019;8: 1-12. doi:10.12688/f1000research.19793.1

33. Schultz W. Open Access REVIEW BioMed Central Dopamine signals for reward value and risk: basic and recent data. 2010; 1-9. doi:10.1186/1744-9081-6-24

34. Heller AS, Johnstone T, Peterson MJ, Kolden GG, Kalin NH, Davidson RJ. Increases in prefrontal cortex activity when regulating negative emotion predicts symptom severity trajectory over six months in depression. JAMA psychiatry. 2013;70: 1181-9. doi:10.1001/JAMAPSYCHIATRY.2013.2430

35. Sharp BM. Basolateral amygdala and stress-induced hyperexcitability affect motivated behaviors and addiction. Transl Psychiatry. 2017;7: e1194. doi:10.1038/tp.2017.161

36. Tomova L, Saxe R, Klöbl M, Lanzenberger R, Lamm C. Acute stress alters neural patterns of value representation for others. Neuroimage. 2020;209. doi:10.1016/j.neuroimage.2019.116497

37. Hecht D. The Neural Basis of Optimism and Pessimism. Exp Neurobiol. 2013;22: 173199. doi:10.5607/en.2013.22.3.173

38. Gruber MJ, Gelman BD, Ranganath C. States of Curiosity Modulate HippocampusDependent Learning via the Dopaminergic Circuit. Neuron. 2014;84: 486-496. doi:10.1016/j.neuron.2014.08.060

39 Hahad O, Kröller-Schön S, Daiber A, Münzel T. Effects of noise on the cardiovascular 
system. Dtsch Arztebl Int. 2019;116. doi:10.3238/arztebl.2019.0245

40. Chevance A, Gourion D, Hoertel N, Llorca PM, Thomas P, Bocher R, et al. Ensuring mental health care during the SARS-CoV-2 epidemic in France: A narrative review. Encephale. 2020. doi:10.1016/j.encep.2020.03.001

41. Pignon B. Dramatic reduction of psychiatric emergency consultations during lockdown linked to COVID- 19 in Paris and suburbs. 2020. doi:10.1101/2020.05.19.20095901

42. Schaller M, Murray DR, Bangerter A. Implications of the behavioural immune system for social behaviour and human health in the modern world. Philos Trans R Soc B Biol Sci. 2015;370. doi:10.1098/rstb.2014.0105

43. Goldfarb EV et al. predicts the feeling of stress. Nat Commun. 2020; 1-10. doi:10.1038/s41467-020-16492-2

44. Lechien JR, Chiesa-Estomba CM, De Siati DR, Horoi M, Le Bon SD, Rodriguez A, et al. Olfactory and gustatory dysfunctions as a clinical presentation of mild-to-moderate forms of the coronavirus disease (COVID-19): a multicenter European study. Eur Arch Otorhinolaryngol. 2020;2. doi:10.1007/s00405-020-05965-1

45. Vaira LA, Salzano G, Deiana G, De Riu G. Anosmia and ageusia: common findings in COVID-19 patients. Laryngoscope. 2020; 1-4. doi:10.1002/lary.28692

46. Gautier J-F, Ravussin Y. A New Symptom of COVID-19: Loss of Taste and Smell. Obesity (Silver Spring). 2020;00: 22809. doi:10.1002/oby.22809

47. Rogers J, Lewis G, London S, Nhs M, Chesney E, Msc O, et al. Psychiatric and neuropsychiatric presentations associated with severe coronavirus infections: a systematic review and meta-analysis with comparison to the COVID-19 pandemic. The Lancet Psychiatry. 2020; 1-17. doi:10.1016/S2215-0366(20)30203-0

48. Troyer EA. Since January 2020 Elsevier has created a COVID-19 resource centre with free information in English and Mandarin on the novel coronavirus COVID- 19. The COVID-19 resource centre is hosted on Elsevier Connect, the company' s public news and information. Brain Behav Immun. 2020.

49. Needham EJ, Chou SHY, Coles AJ, Menon DK. Neurological implications of COVID19 infections (submitted). Lancet Neurol. 2020. doi:10.1007/s12028-020-00978-4

50. Figueroa G. Neuroethics: The pursuit of transforming medical ethics in scientific ethics. Biol Res. 2016;49: 1-7. doi:10.1186/s40659-016-0070-y

51. Darragh M, Buniak L, Giordano J. A four-part working bibliography of neuroethics: Part 2 - neuroscientific studies of morality and ethics. Philos Ethics, Humanit Med. 2015;10: 1-22. doi:10.1186/s13010-015-0022-0

52. Martin A, Becker K, Darragh M, Giordano J. A four-part working bibliography of neuroethics: Part 3 - "second tradition neuroethics" - ethical issues in neuroscience. 
Philosophy, Ethics, and Humanities in Medicine. Philosophy, Ethics, and Humanities in Medicine; 2016. doi:10.1186/s13010-016-0037-1

53. Becker K, Shook JR, Darragh M, Giordano J. A four-part working bibliography of neuroethics: Part 4 - Ethical issues in clinical and social applications of neuroscience. Philosophy, Ethics, and Humanities in Medicine. Philosophy, Ethics, and Humanities in Medicine; 2017. doi:10.1186/s13010-017-0043-y

54. Ramos KM, Grady C, Greely HT, Chiong W, Eberwine J, Farahany NA, et al. The NIH BRAIN Initiative: Integrating Neuroethics and Neuroscience. Neuron. 2019;101: 394-398. doi:10.1016/j.neuron.2019.01.024

55. Kennett J, Carter A, Bourne JA, Hall W, Levy N, Mattingley JB, et al. A Neuroethics Framework for the Australian Brain Initiative. Neuron. 2019;101: 365-369. doi:10.1016/j.neuron.2019.01.004

56. Jeong SJ, Lee IY, Jun BO, Ryu YJ, Sohn J woo, Kim SP, et al. Korea Brain Initiative: Emerging Issues and Institutionalization of Neuroethics. Neuron. 2019;101: 390-393. doi:10.1016/j.neuron.2019.01.042

57. Amunts K, Knoll AC, Lippert T, Pennartz CMA, Ryvlin P, Destexhe A, et al. The Human Brain Project-Synergy between neuroscience, computing, informatics, and brain-inspired technologies. PLoS Biol. 2019;17: 1-7. doi:10.1371/journal.pbio.3000344

58. Rose N. The human brain project: Social and ethical challenges. Neuron. 2014;82: 1212-1215. doi:10.1016/j.neuron.2014.06.001

59. Salles A, Bjaalie JG, Evers K, Farisco M, Fothergill BT, Guerrero M, et al. The Human Brain Project: Responsible Brain Research for the Benefit of Society. Neuron. 2019;101: 380-384. doi:10.1016/j.neuron.2019.01.005

60. Kögel J, Schmid JR, Jox RJ, Friedrich O. Using brain-computer interfaces: A scoping review of studies employing social research methods. BMC Med Ethics. 2019;20: 1517. doi:10.1186/s12910-019-0354-1

61. Shen FX, Wolf SM, Gonzalez RG, Garwood M. Ethical Issues Posed by Field Research Using Highly Portable and Cloud-Enabled Neuroimaging. Neuron. 2020;105: 771-775. doi:10.1016/j.neuron.2020.01.041

62. Nadelhoffer T, Bibas S, Grafton S, Kiehl KA, Mansfield A, Sinnott-Armstrong W, et al. Neuroprediction, violence, and the law: Setting the stage. Neuroethics. 2012;5: 6799. doi:10.1007/s12152-010-9095-z

63. Tortora L, Meynen G, Bijlsma J, Tronci E, Ferracuti S. Neuroprediction and A.I. in Forensic Psychiatry and Criminal Justice: A Neurolaw Perspective. Front Psychol. 2020;11: 1-9. doi:10.3389/fpsyg.2020.00220 
64. Steinert S, Friedrich O. Wired Emotions: Ethical Issues of Affective Brain-Computer Interfaces. Sci Eng Ethics. 2020;26: 351-367. doi:10.1007/s11948-019-00087-2

65. Minielly N, Hrincu V, Illes J. Privacy Challenges to the Democratization of Brain Data. iScience. 2020;23: 101134. doi:10.1016/j.isci.2020.101134

66. Bannai H, Niwa F, Sherwood MW, Shrivastava AN, Arizono M, Miyamoto A, et al. Bidirectional Control of Synaptic GABAAR Clustering by Glutamate and Calcium. Cell Rep. 2015;13: 2768-2780. doi:10.1016/j.celrep.2015.12.002

67. Mapelli J, Gandolfi D, Vilella A, Zoli M, Bigiani A. Heterosynaptic GABAergic plasticity bidirectionally driven by the activity of pre- and postsynaptic NMDA receptors. Proc Natl Acad Sci U S A. 2016;113: 9898-9903. doi:10.1073/pnas.1601194113

68. Ito Hiroyuki. Self-reorganization of neuronal activation patterns in the cortex under brain-machine interface and neural operant conditioning. Neurosci Res. 2020;20: 30174-7. doi:https://doi.org/10.1016/j.neures.2020.03.008

69. Lajoie G, Krouchev NI, Kalaska JF, Fairhall AL, Fetz EE. Correlation-based model of artificially induced plasticity in motor cortex by a bidirectional brain-computer interface. PLoS Computational Biology. 2017. doi:10.1371/journal.pcbi.1005343

70. Zheng Y, Mao YR, Yuan TF, Xu DS, Cheng LM. Multimodal treatment for spinal cord injury: A sword of neuroregeneration upon neuromodulation. Neural Regen Res. 2020;15: 1437-1450. doi:10.4103/1673-5374.274332

71. Ajiboye AB, Willett FR, Young DR, Memberg WD, Murphy BA, Miller JP, et al. Restoration of reaching and grasping movements through brain-controlled muscle stimulation in a person with tetraplegia: a proof-of-concept demonstration. Lancet. 2017;389: 1821-1830. doi:10.1016/S0140-6736(17)30601-3

72. Vachez Yvan. Subthalamic Nucleus Stimulation Impairs Motivation: Implication for Apathy in Parkinson's Disease. Mov Disord. 2020;35: 616-628. doi:https://doi.org/10.1002/mds.27953

73. Irmen Friederike. Left prefrontal impact links subthalamic stimulation with depressive symptoms. Ann Neurol. 2020;87: 962-975. doi:https://doi.org/10.1002/ana.25734

74. Munyon CN. Neuroethics of Non-primary Brain Computer Interface: Focus on Potential Military Applications. Front Neurosci. 2018;12: 10-13. doi:10.3389/fnins.2018.00696

75. Marcello Ienca KI. Artificial Intelligence in Clinical Neuroscience: Methodological and Ethical Challenges. AJOB Neurosci. 2020;11: 77-87. doi:https://doi.org/10.1080/21507740.2020.1740352

76. Lindsay GW. Attention in Psychology, Neuroscience, and Machine Learning. Front 
Comput Neurosci. 2020;14: 1-21. doi:10.3389/fncom.2020.00029

77. Rieder T. Artificial Intelligence in Service of Human Needs: Pragmatic First Steps Toward an Ethics for Semi-Autonomous Agents. AJOB Neurosci. 2020;11: 120-127. doi:10.1080/21507740.2020.1740354

78. Mittelstadt B. Principles alone cannot guarantee ethical AI. Nat Mach Intell. 2019;1: 501-507. doi:10.1038/s42256-019-0114-4

79. Frewen P, Schroeter ML, Riva G, Cipresso P, Fairfield B, Padulo C, et al. When Thinking is Doing: Responsibility for BCI-Mediated Action. Neurosci Biobehav Rev. 2020;14: 101134. doi:10.1007/s11948-020-00218-0

80. Rainey S, Maslen H, Savulescu J. When Thinking is Doing: Responsibility for BCIMediated Action. AJOB Neurosci. 2020;11: 46-58. doi:10.1080/21507740.2019.1704918 